\title{
Pacific
}

Journal of

Mathematics

\section{ON QUADRATIC RECIPROCITY OVER FUNCTION FIELDS}

Kathy DONOVAn MERRILl AND LyNNe WaLLing

Volume 173 No. 1

March 1996 


\title{
ON QUADRATIC RECIPROCITY OVER FUNCTION FIELDS
}

\author{
Kathy D. Merrill and Lynne H. Walling
}

\begin{abstract}
A proof of quadratic reciprocity over function fields is given using the inversion formula of the theta function.
\end{abstract}

Over the years, many authors have produced proofs of the law of quadratic reciprocity. In 1857, Dedekind [2] stated that quadratic reciprocity holds over function fields; this was later proved by Artin [1]. One of the simplest proofs over the rational numbers relies on the functional equation of the classical theta function (see, for example, [3]); this technique was later generalized by Hecke [4] to number fields. In this note we use an analogous technique to give a simple and direct proof of quadratic reciprocity over rational function fields. We thank David Grant for suggesting this application of Theorem 2.3 of $[\mathbf{6}]$.

The reader is referred to [5] for a more complete discussion of the history of the Law of Quadratic Reciprocity.

Let $\mathbf{F}=\mathbf{F}_{p}$ be a finite field with $p$ elements; for the sake of clarity we assume $p$ is an odd prime. Let $T$ be an indeterminate, and set $\mathbf{A}=\mathbf{F}[T]$. Then for $\alpha, \beta \in \mathbf{A}$ with $\alpha$ irreducible, let

$$
\left(\frac{\beta}{\alpha}\right)= \begin{cases}1 & \text { if } \beta \text { is a (nonzero) quadratic residue modulo } \alpha, \\ -1 & \text { if } \beta \text { is a (nonzero) quadratic nonresidue modulo } \alpha \\ 0 & \text { if } \alpha \text { divides } \beta .\end{cases}
$$

We will show that for $\alpha, \beta \in \mathbf{A}$ distinct monic irreducible polynomials,

$$
\left(\frac{\beta}{\alpha}\right)= \begin{cases}\left(\frac{-1}{p}\right)\left(\frac{\alpha}{\beta}\right) & \text { if } \operatorname{deg} \alpha, \operatorname{deg} \beta \text { are both odd } \\ \left(\frac{\alpha}{\beta}\right) & \text { otherwise. }\end{cases}
$$

We require the following definitions.

Let $\mathbf{K}=\mathbf{F}(T)$; let $\mathbf{K}_{\infty}$ denote the completion of $\mathbf{K}$ with respect to the "infinite" valuation $|\cdot|_{\infty}$ given by $|\alpha / \beta|_{\infty}=p^{\operatorname{deg} \alpha-\operatorname{deg} \beta}$ where $\alpha, \beta \in \mathbf{A}$. (We adopt the convention that $\operatorname{deg} 0=-\infty$, and hence $|0|_{\infty}=0$.) One easily sees that $\mathbf{K}_{\infty}=\mathbf{F}\left(\left(\frac{1}{T}\right)\right)$, formal Laurent series in $\frac{1}{T}$; for $x \in \mathbf{K}_{\infty}$, we write $x=\sum_{j=-\infty}^{n} x_{j} T^{j}$. The "unit ball" or "ring of integers" in $\mathbf{K}_{\infty}$ is 
$\mathcal{O}_{\infty}=\left\{x \in \mathbf{K}_{\infty}:|x|_{\infty} \leq 1\right\}=\mathbf{F}\left[\left[\frac{1}{T}\right]\right]$, formal Taylor series in $\frac{1}{T}$. Set $G=P S L_{2}\left(\mathbf{K}_{\infty}\right)$; then the maximal compact subgroup of $G$ (with respect to the standard topology induced on $G$ by $\left.|\cdot|_{\infty}\right)$ is $P S L_{2}\left(\mathcal{O}_{\infty}\right)$. Thus we set

$$
\mathbf{H}=P S L_{2}\left(\mathbf{K}_{\infty}\right) / P S L_{2}\left(\mathcal{O}_{\infty}\right) \text {. }
$$

We can view $P S L_{2}(*)$ as a subgroup of $P G L_{2}(*)$; so we consider a matrix of $P S L_{2}(*)$ equivalent to every nonzero scalar multiple of the matrix. Then as shown in [6],

$$
\left\{\left(\begin{array}{ll}
y & x \\
0 & 1
\end{array}\right): y=T^{2 m}, m \in \mathbf{Z}, x \in T^{2 m+1} \mathbf{A}\right\}
$$

is a complete set of representatives for $\mathbf{H}$. For each $z \equiv\left(\begin{array}{ll}y & x \\ 0 & 1\end{array}\right) \in \mathbf{H}$, set

$$
\theta(z)=\sum_{\delta \in \mathbf{A}} \chi_{\mathcal{O}_{\infty}}\left((T \delta)^{2} y\right) e\left\{(T \delta)^{2} x\right\}
$$

where $e\{\gamma\}=e\left\{\sum_{j \geq N} \gamma_{j} T^{j}\right\}=\exp \left(2 \pi i \gamma_{1} / p\right)$ and $\chi_{\mathcal{O}_{\infty}}$ is the characteristic function for $\mathcal{O}_{\infty}$.

As in the classical setting, we will connect this theta series to quadratic reciprocity through Gauss sums. Accordingly, for $\alpha, \beta \in \mathbf{A}$ with $\alpha$ irreducible and $\alpha$ not dividing $\beta$, define the Gauss sum $G_{\alpha}(\beta)$ to be $G_{\alpha}(\beta)=$ $\sum_{\delta \in \mathbf{A} / \alpha \mathbf{A}} e\left\{\beta \delta^{2} T^{2} / \alpha\right\}$.

Lemma 1. For $\alpha, \beta \in \mathbf{A}$ with $\alpha$ irreducible and $\alpha \not \beta,\left(\frac{\beta}{\alpha}\right)=\frac{G_{\alpha}(\beta)}{G_{\alpha}(1)}$. Proof. We have

$$
G_{\alpha}(\beta)=\sum_{\delta \in \mathbf{A} / \alpha \mathbf{A}}\left(1+\left(\frac{\delta}{\alpha}\right)\right) e\left\{\beta \delta T^{2} / \alpha\right\}=\sum_{\delta \in \mathbf{A}}\left(\frac{\delta}{\alpha}\right) e\left\{\beta \delta T^{2} / \alpha\right\}
$$

and for $\beta^{\prime} \in \mathbf{A}$ such that $\beta \beta^{\prime} \equiv 1(\bmod \alpha)$

$$
=\sum_{\delta \in \mathbf{A} / \alpha \mathbf{A}}\left(\frac{\delta \beta^{\prime}}{\alpha}\right) e\left\{\beta \delta \beta^{\prime} T^{2} / \alpha\right\}=\left(\frac{\beta^{\prime}}{\alpha}\right) G_{\alpha}(1)=\left(\frac{\beta}{\alpha}\right) G_{\alpha}(1) .
$$

Lemma 2. For $\alpha, \beta$ relatively prime irreducible polynomials, $G_{\alpha}(\beta) G_{\beta}(\alpha)=$ $G_{\alpha \beta}(1)$.

Proof. Notice that the map $(\delta+\alpha \beta \mathbf{A}, \gamma+\alpha \beta \mathbf{A}) \mapsto \delta+\gamma+\alpha \beta \mathbf{A}$ is an injective homomorphism from $(\beta \mathbf{A} / \alpha \beta \mathbf{A}) \times(\alpha \mathbf{A} / \alpha \beta \mathbf{A})$ into $\mathbf{A} / \alpha \beta \mathbf{A}$; since the cardinalities of the domain and the codomain are finite and equal, the map is an 
isomorphism. Also notice that for $\delta \in \beta \mathbf{A}$ and $\gamma \in \alpha \mathbf{A}, e\left\{(\delta+\gamma)^{2} T^{2} / \alpha \beta\right\}=$ $e\left\{\delta^{2} T^{2} / \alpha \beta\right\} e\left\{\gamma^{2} T^{2} / \alpha \beta\right\}$. Thus

$$
G_{\alpha \beta}(1)=\sum_{\delta \in \mathbf{A} / \alpha \mathbf{A}} e\left\{(\beta \delta)^{2} T^{2} / \alpha \beta\right\} \sum_{\gamma \in \mathbf{A} / \beta \mathbf{A}} e\left\{(\alpha \delta)^{2} T^{2} / \alpha \beta\right\}=G_{\alpha}(\beta) G_{\beta}(\alpha) .
$$

Combining these two lemmata, we have that for $\alpha, \beta$ relatively prime irreducible polynomials, $\left(\frac{\alpha}{\beta}\right)\left(\frac{\beta}{\alpha}\right)=\frac{G_{\alpha \beta}(1)}{G_{\alpha}(1) G_{\beta}(1)}$. Thus for formulate the law of Quadratic Reciprocity, we need only evaluate $G_{\gamma}(1)$ for $\alpha \in \mathbf{A}$. This is the content of our final lemma.

Lemma 3. For any $\gamma \in \mathbf{A}, G_{\gamma}(1)=p^{\frac{d}{2}}\left(\frac{\gamma_{d}}{p}\right)^{d} \sqrt{\left(\frac{-1}{p}\right)^{d}}$ where $d=\operatorname{deg} \gamma$ and $\gamma_{d}$ denotes the coefficient of $T^{d}$ in $\gamma$.

Proof. First notice that by the Euclidean Algorithm, $\{\delta \in \mathbf{A}: \operatorname{deg} \delta<d\}$ is a complete set of representatives for $\mathbf{A} / \gamma \mathbf{A}$. Thus

$$
G_{\gamma}(1)=\sum_{\delta \in \mathbf{A}} \chi_{\mathcal{O}_{\infty}}\left((T \delta)^{2} T^{-2 d}\right) e\left\{(T \delta)^{2} / \gamma\right\}
$$

Letting $z=\left(\begin{array}{cc}T^{-2 d} & \frac{1}{\gamma} \\ 0 & 1\end{array}\right)$, we see that $G_{\gamma}(1)=\theta(z)$ where $\theta(z)$ is as in $[\mathbf{6}]$. By the Inversion Formula, we have $\theta(z)=p^{\frac{d}{2}}\left(\frac{\gamma_{d}}{p}\right)^{d} \sqrt{\left(\frac{-1}{p}\right)^{d}} \theta\left(-\frac{1}{z}\right)$ where $-\frac{1}{z}=\left(\begin{array}{cc}0 & -1 \\ 1 & 0\end{array}\right) z \equiv\left(\begin{array}{cc}1 & -\gamma \\ 0 & 1\end{array}\right)$. Since the only $\delta \in \mathbf{A}$ satisfying $\chi_{\mathcal{O}_{\infty}}\left((T \delta)^{2}\right)=$ 1 is $\delta=0, \theta\left(-\frac{1}{z}\right)=1$.

These Lemmata easily imply the following

Theorem. Let $\alpha, \beta$ be relatively prime irreducible polynomials of degrees $d$ and $d^{\prime}$ respectively. Then

$$
\left(\frac{\alpha}{\beta}\right)=\epsilon\left(\frac{\alpha_{d}}{p}\right)^{d^{\prime}}\left(\frac{\beta_{d^{\prime}}}{p}\right)^{d}\left(\frac{\beta}{\alpha}\right)
$$

where

$$
\epsilon= \begin{cases}\left(\frac{-1}{p}\right) & \text { if } d, d^{\prime} \text { are both odd } \\ 1 & \text { otherwise }\end{cases}
$$


In particular, when $\alpha$ and $\beta$ are distinct monic irreducible polynomials,

$$
\left(\frac{\alpha}{\beta}\right)= \begin{cases}\left(\frac{-1}{p}\right)\left(\frac{\beta}{\alpha}\right) & \text { if } d, d^{\prime} \text { are both odd } \\ \left(\frac{\beta}{\alpha}\right) & \text { otherwise. }\end{cases}
$$

\section{References}

[1] E. Artin, Quadratische Körper im Gebiete der höheren Kongruenzen, Math. Zeit., 19 (1924), 153-246.

[2] R. Dedekind, Abriss einer Theorie der höheren Congruenzen in Bezug auf einer reellen Primzahl-Modulus, J. reine und angew. Math., 54 (1857), 1-26.

[3] H. Dym and H.P. McKean, Fourier Series and Integrals, Academic Press, New York, 1972.

[4] E. Hecke, Lectures on the Theory of Algebraic Numbers, Springer-Verlag, New YorkHeidelberg-Berlin, 1981.

[5] K. Ireland and M. Rosen, A Classical Introduction to Modern Number Theory, Springer-Verlag, New York-Berlin-Heidelberg-London-Paris-Tokyo-Hong Kong, 1990.

[6] K.D. Merrill and L.H. Walling, Sums of squares over function fields, Duke Math. J., 71(3) (1993), 665-684.

Received September 27, 1993 and revised March 25, 1994. The second author was partially supported by NSF grant DMS 9103303.

Colorado College

Colorado Springs, CO 80903

E-mail address: merrill@cc.colorado.edu

AND

UNIVERSITY OF COLORADO

BOULDER, CO 80309-0426

E-mail address: walling@euclid.colorado.edu 


\title{
PACIFIC JOURNAL OF MATHEMATICS
}

Founded by

\author{
$\begin{array}{ll}\text { E. F. Beckenbach (1906-1982) F. Wolf (1904-1989) } & \text { F }\end{array}$
}

EDITORS

Sun-Yung A. Chang (Managing Editor) Robert Finn

University of California

Los Angeles, CA 90095-1555

pacific@math.ucla.edu

F. Michael Christ

University of California

Los Angeles, CA 90095-1555

christ@math.ucla.edu

Nicholas Ercolani

University of Arizona

Tucson, AZ 85721

ercolani@math.arizona.edu
Stanford University

Stanford, CA 94305

finn@gauss.stanford.edu

Steven Kerckhoff

Stanford University

Stanford, CA 94305

spk@gauss.stanford.edu

Martin Scharlemann

University of California

Santa Barbara, CA 93106

mgscharl@math.ucsb.edu
Gang Tian

Massachusettes Institute of Technology

Cambridge, MA 02139

tian@math.mit.edu

\section{S. Varadarajan}

University of California

Los Angeles, CA 90095-1555

vsv@math.ucla.edu

Dan Voiculescu

University of California

Berkeley, CA 94720

dvv@math.berkeley.edu

\section{SUPPORTING INSTITUTIONS}

\author{
CALIFORNIA INSTITUTE OF TECHNOLOGY \\ NEW MEXICO STATE UNIVERSITY \\ OREGON STATE UNIVERSITY \\ STANFORD UNIVERSITY \\ UNIVERSITY OF ARIZONA \\ UNIVERSITY OF BRITISH COLUMBIA \\ UNIVERSITY OF CALIFORNIA \\ UNIVERSITY OF HAWAII
}

\author{
UNIVERSITY OF MONTANA \\ UNIVERSITY OF NEVADA, RENO \\ UNIVERSITY OF OREGON \\ UNIVERSITY OF SOUTHERN CALIFORNIA \\ UNIVERSITY OF UTAH \\ UNIVERSITY OF WASHINGTON \\ WASHINGTON STATE UNIVERSITY
}

The supporting Institutions listed above contribute to the cost of publication of this Journal, but they are not owners or publishers and have no responsibility for its contents or policies.

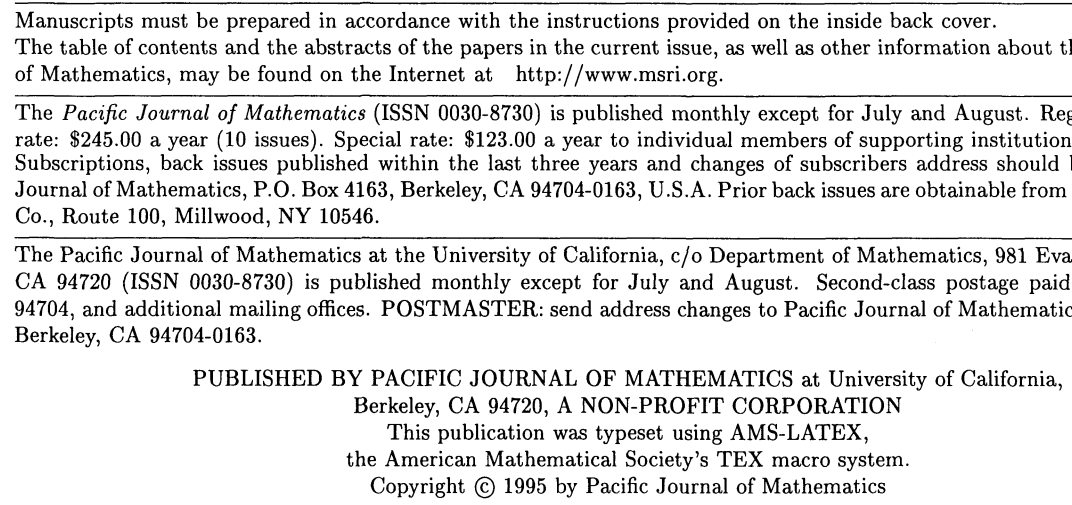




\section{PACIFIC JOURNAL OF MATHEMATICS}

\section{Volume $173 \quad$ No. $1 \quad$ March 1996}

Isometric immersions of $H_{1}^{n}$ into $H_{1}^{n+1}$

KINETSU ABE

Rotationally symmetric hypersurfaces with prescribed mean curvature

MARIE-FRANÇOISE BIDAUT-VÉRON

The covers of a Noetherian module

JIAN-JUN CHUAI

On the odd primary cohomology of higher projective planes

MARK FoSKEY and MiCHAEL DAVID SLACK

Unit indices of some imaginary composite quadratic fields. II

MiKinito HiRABAYASHI

Mixed automorphic vector bundles on Shimura varieties

MIN Ho LeE

Trace ideal criteria for Toeplitz and Hankel operators on the weighted Bergman

spaces with exponential type weights

PENG LIN and RICHARD ROCHBERG

On quadratic reciprocity over function fields

KATHY DONOVAN MERRILL and LYNNE WALLING

$\left(A_{2}\right)$-conditions and Carleson inequalities in Bergman spaces

TAKAHIKO NAKAZI and MASAHIRO YAMADA

A note on a paper of E. Boasso and A. Larotonda: "A spectral theory for solvable Lie 173 algebras of operators"
C. Отт

Tensor products with anisotropic principal series representations of free groups

Carlo Pensavalle and Tim Steger

On Ricci deformation of a Riemannian metric on manifold with boundary

YING SHEN

The Weyl quantization of Poisson $S U(2)$

Albert Jeu-Liang Sheu

Weyl's law for $S L(3, \mathbb{Z}) \backslash S L(3, \mathbb{R}) / S O(3, \mathbb{R})$

ERIC GeORge Stade and Dorothy IRENe Wallace (ANDREOli)

Minimal hyperspheres in two-point homogeneous spaces

PER TOMTER

Subalgebras of little Lipschitz algebras

NiKOLAI ISAAC WEAVER 